\title{
Fractura de odontoides: tratamiento, pronóstico y morbimortalidad asociada en un Hospital de Alta Concentración
}

\author{
Odontoid fracture: treatment, prognosis and associated \\ morbimortality in a High Concentration Hospital
}

Jorge Andrés Esparza Piña*

*Ortopedia y Traumatología, Clínica de Columna. Centro Médico Nacional de Occidente, Unidad Médica de Alta Especialidad. IMSS.

\begin{abstract}
Resumen
Introducción: La apófisis odontoides o proceso odontoideo es un elemento óseo superior, sobresaliente del cuerpo de la segunda vértebra cervical. El atlas gira alrededor del proceso odontoideo para proporcionar el componente único más grande de rotación lateral de la columna cervical. Las fracturas de odontoides son fracturas relativamente frecuentes del cuerpo vertebral de C2 que se pueden ver en caídas de baja energía en pacientes de edad avanzada y en lesiones traumáticas de alta energía en pacientes jóvenes. Objetivo: Analizar la morbimortalidad asociada, pronóstico y tratamiento de pacientes con fractura de apófisis odontoides en el occidente en un hospital de alta concentración y actualidades de tratamiento. Material y métodos: Diseño de estudio: casos y controles. Análisis retrospectivo con diagnóstico de fractura de apófisis odontoides tipo II. Se evaluó tratamiento conservador vs quirúrgico fijación anterior o instrumentación posterior. Para variables cualitativas se utilizaron frecuencias y porcentajes. Para variables cuantitativas se emplearon medias y desviaciones estándar. Estadística inferencial: Prueba $\chi^{2}$ y Fisher. Todo valor de $p$. Resultados: Se identificaron 29 pacientes que cumplieron con los criterios de inclusión, de los cuales $93.1 \%$ (27 pacientes) fueron clasificados como Anderson y D'Alonzo tipo II y $6.9 \%$ (dos pacientes) como Anderson y D'Alonzo tipo III. La edad promedio de los pacientes fue de $50.93 \pm 20.90$ años. Conclusiones: El tratamiento quirúrgico en los pacientes con fractura de odontoides presenta un mayor índice de éxito en la resolución de los pacientes con dicha patología mas no modifica el índice de morbimortalidad asociada.
\end{abstract}

Palabras clave: Proceso odontoide, fractura C2, eje, Anderson y D'Alonzo, fractura odontoide de tipo II.

\begin{abstract}
Introduction: The odontoid process is a superior bone element, protruding from the body of the second cervical vertebra C2. The atlas rotates around the odontoid process to provide the single largest component of lateral rotation of the cervical spine. Odontoid fractures are relatively common fractures of the C2 vertebral body that can be seen in low-energy falls in older patients and in highenergy traumatic injuries in young patients. Objective: To analyze the associated morbidity and mortality, prognosis and treatment of patients with fracture of the odontoid process in the West in a high-concentration hospital and treatment updates. Material and methods: Study design: cases and controls. Retrospective analysis with diagnosis of type II odontoid process fracture. Conservative treatment vs. surgical anterior fixation or posterior instrumentation is evaluated. For qualitative variables, frequencies and percentages are used. For quantitative variables, means and standard deviations will be used. Inferential statistics: $\chi^{2}$ and fisher test. Any value of p. Results: 29 patients were identified who met the inclusion criteria, of which 93.1\% (27 patients) were classified as Anderson and D'Alonzo type II and 6.9\% (two patients) as Anderson and D'Alonzo type III. The mean age of the patients was $50.93 \pm 20.90$ years. Conclusions: Surgical treatment in patients with odontoid fracture has a higher rate of success in the resolution of patients with this pathology but does not modify the associated morbidity and mortality rate.
\end{abstract}

Keywords: Odontoid process, C2 fracture, axis, Anderson and D’Alonzo, type II odontoid fracture.

Correspondencia:

Jorge Andrés Esparza Piña

E-mail: jandres.esparza@gmail.com

Recibido: 10-02-2021. Aceptado: 15-02-2021.

Citar como: Esparza PJA. Fractura de odontoides: tratamiento, pronóstico y morbimortalidad asociada en un Hospital de Alta Concentración. Orthotips. 2021; 17 (1): 18-24. https://dx.doi.org/10.35366/99163 


\section{Introducción}

La apófisis odontoides o proceso odontoideo es un elemento óseo superior, sobresaliente del cuerpo de la segunda vértebra cervical (C2), el atlas (C1) gira alrededor del proceso odontoideo para proporcionar el componente más grande de rotación de la columna cervical. ${ }^{1}$

Las fracturas de odontoides son relativamente frecuentes del cuerpo vertebral de $\mathrm{C} 2$ que se pueden ver en caídas de baja energía en pacientes de edad avanzada y en lesiones traumáticas de alta energía en pacientes jóvenes. El mecanismo de lesión más común es una hiperextensión de la columna cervical, si el mecanismo de alta energía y fuerza resultante, o la densidad ósea del paciente se encuentra disminuida, se presentará una fractura de odontoides con diferentes desplazamientos y grados de conminución. ${ }^{1}$

La fractura odontoidea también puede ocurrir con hiperflexión de la columna cervical, si la columna cervical está excesivamente flexionada, el ligamento transversal puede transmitir las fuerzas anteriores excesivas al proceso odontoideo y causar una fractura odontoidea, ${ }^{2}$ el tipo más común de fractura de C2 es la fractura de la apófisis odontoides. ${ }^{3}$

Las fracturas de odontoides muestran una distribución bifásica con tasas de incidencia máxima notificadas en pacientes más jóvenes (de 20 a 30 años) secundarios a mecanismos de alta energía, seguidos de poblaciones de pacientes de edad avanzada (de 70 a 80 años) secundarias a densidad ósea comprometida y caída de propia altura de bajo impacto energético. ${ }^{3}$

La incidencia de lesiones traumáticas de la médula espinal en los Estados Unidos es mayor a 11,000 casos por año, alrededor de $60 \%$ de las lesiones de la columna vertebral afectan a la columna cervical y aproximadamente uno de cada cinco casos de todas las lesiones de la columna cervical involucra C2, siendo en ésta la lesión más frecuente la fractura odontoidea, de las cuales la mayoría son fracturas de tipo II y representan más de $50 \%$ de todas las fracturas de odontoides. ${ }^{4}$

Las fracturas de odontoides representan de 10 a $15 \%$ de todas las fracturas de columna cervical, siendo la tipo III la mayor parte del porcentaje restante de éstas y las tipo I son raras. ${ }^{5}$

Con menos frecuencia el paciente puede tener lesiones asociadas a mielopatía cervical; ${ }^{6}$ en atención hospitalaria de primer contacto las radiografías son primordiales, se solicitan proyecciones cervica- les anteroposterior, transoral, lateral, oblicuas y en ciertas indicaciones radiografías dinámicas en flexoextensión. El estudio de imagen de elección es una tomografía axial computarizada (TAC) cervical, la cual proporciona la mejor resolución de los elementos óseos, lo que permite la identificación y clasificación de una fractura de apófisis odontoides. Si hay una lesión neurológica se debe obtener una resonancia magnética de columna cervical para lesiones asociadas, en algunos casos también pudiera llegar a solicitarse una angiotomografía. ${ }^{6}$

El tratamiento dependerá de la ubicación de la fractura de odontoides, según lo descrito por la clasificación de Anderson y D’Alonzo y los factores de riesgo del paciente para la falta de unión (la edad del paciente y sus comorbilidades) (Figuras 1 y 2). ${ }^{7} \mathrm{La}$ osteosíntesis de las fracturas de la apófisis odontoides es un desafío debido a la anatomía y a las estructuras vecinas críticas que hacen que la fijación quirúrgica sea una tarea exigente. ${ }^{6,8}$

El objetivo de este estudio es analizar la morbimortalidad asociada, pronóstico y tratamiento de pacientes con fractura de apófisis odontoides en el occidente de México con casos reportados en un hospital de alta concentración.

\section{Material y métodos}

Se trata de un diseño de casos y controles con diagnóstico de fractura de apófisis odontoides en el que se evaluó el manejo conservador vs técnica quirúrgica (fijación anterior o instrumentación posterior). Se incluyeron a pacientes con fractura de odontoides Anderson y D'Alonzo tipo II y III con edades entre los 18 y 80 años; se excluyeron a pacientes con fractura de odontoides Anderson y D'Alonzo tipo I, con inestabilidad hemodinámica previa con traumatismo craneoencefálico severo que fueron sometidos a cirugía craneal descompresiva, y se eliminaron a los pacientes que por razones ajenas a este protocolo solicitaron su alta voluntaria, fallecieron previo a la cirugía o pacientes que presentan Os odontoideum o alguna variedad anatómica odontoides comentada.

Se realizó una estadística descriptiva, para las variables cualitativas se valoraron frecuencias absolutas y relativas y, para variables cuantitativas, se utilizaron medias y desviaciones estándar.

En la estadística inferencial para variables cualitativas se utilizó la prueba $\chi^{2}$ y cuando los valores fueron menores de cinco se utilizó la prueba exacta de Fisher, todo valor de $p$. 

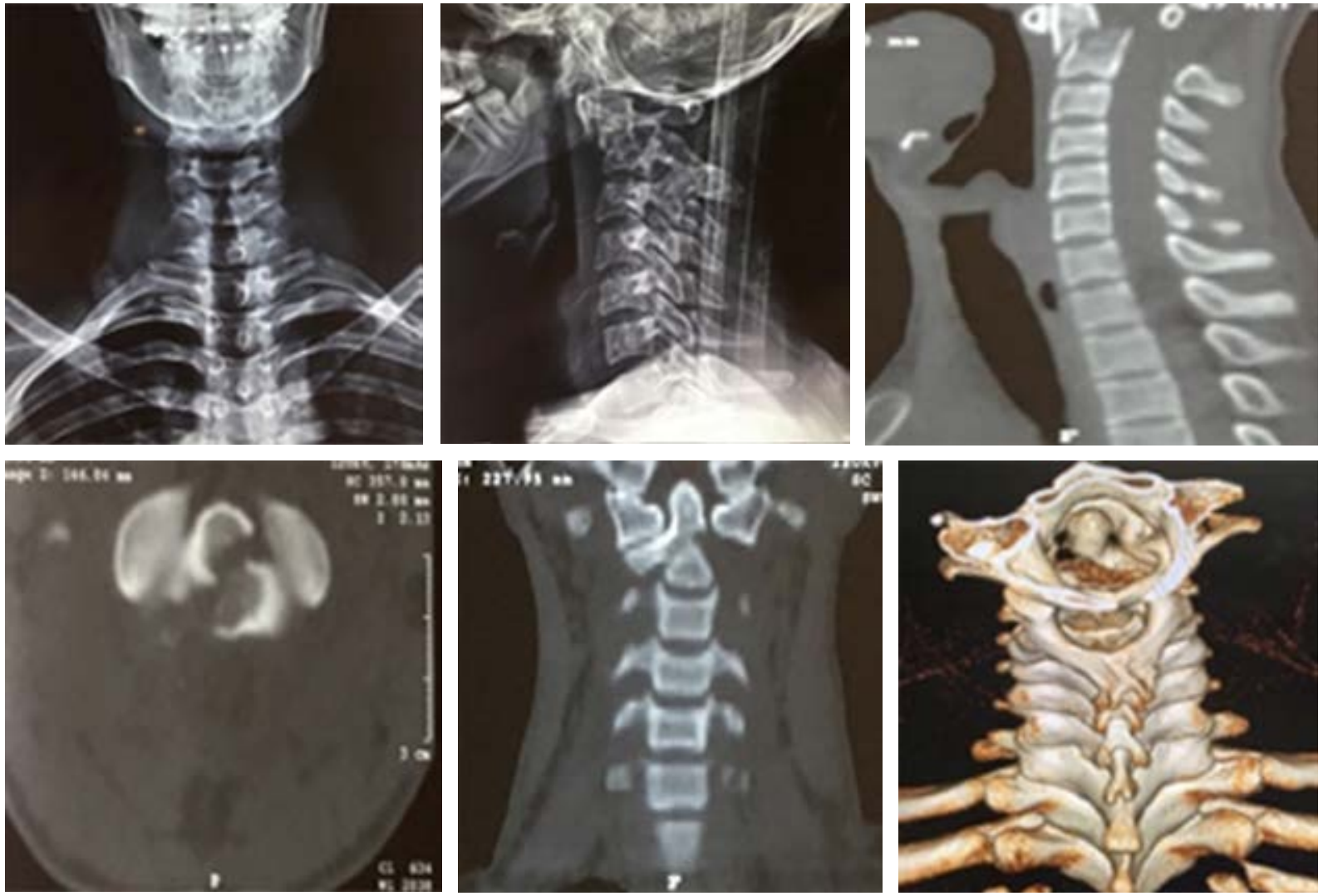

Figura 1: Paciente masculino de 21 años, quien presenta mecanismo de alta energía tras accidente en motocicleta con fractura de tibia asociada. Fractura de apófisis odontoides tipo II Anderson y D'Alonzo, Grauer IIC con desplazamiento mayor a 5 mm.
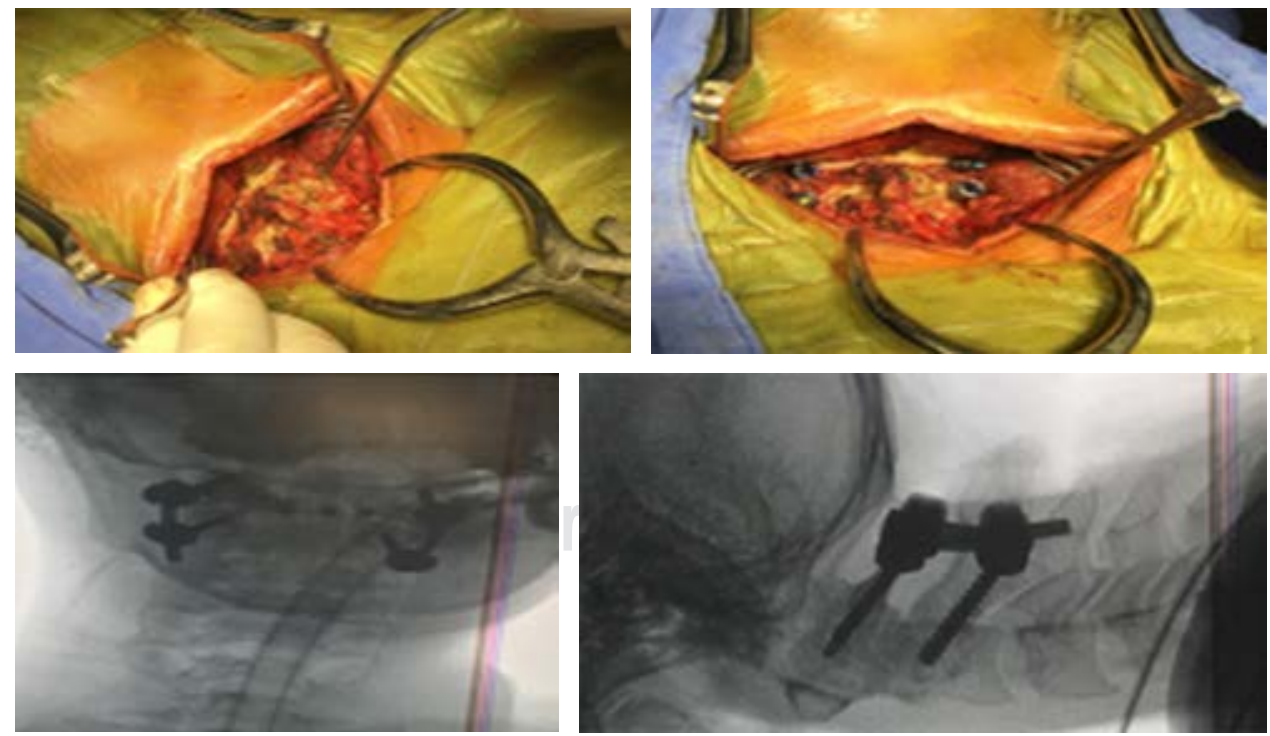

Figura 2: Se realiza manejo quirúrgico instrumentación posterior C1-C2 sin fusión tras reducción cerrada con apoyo de halo cefálico, control fluoroscópico transquirúrgico con adecuada reducción y estabilidad. 

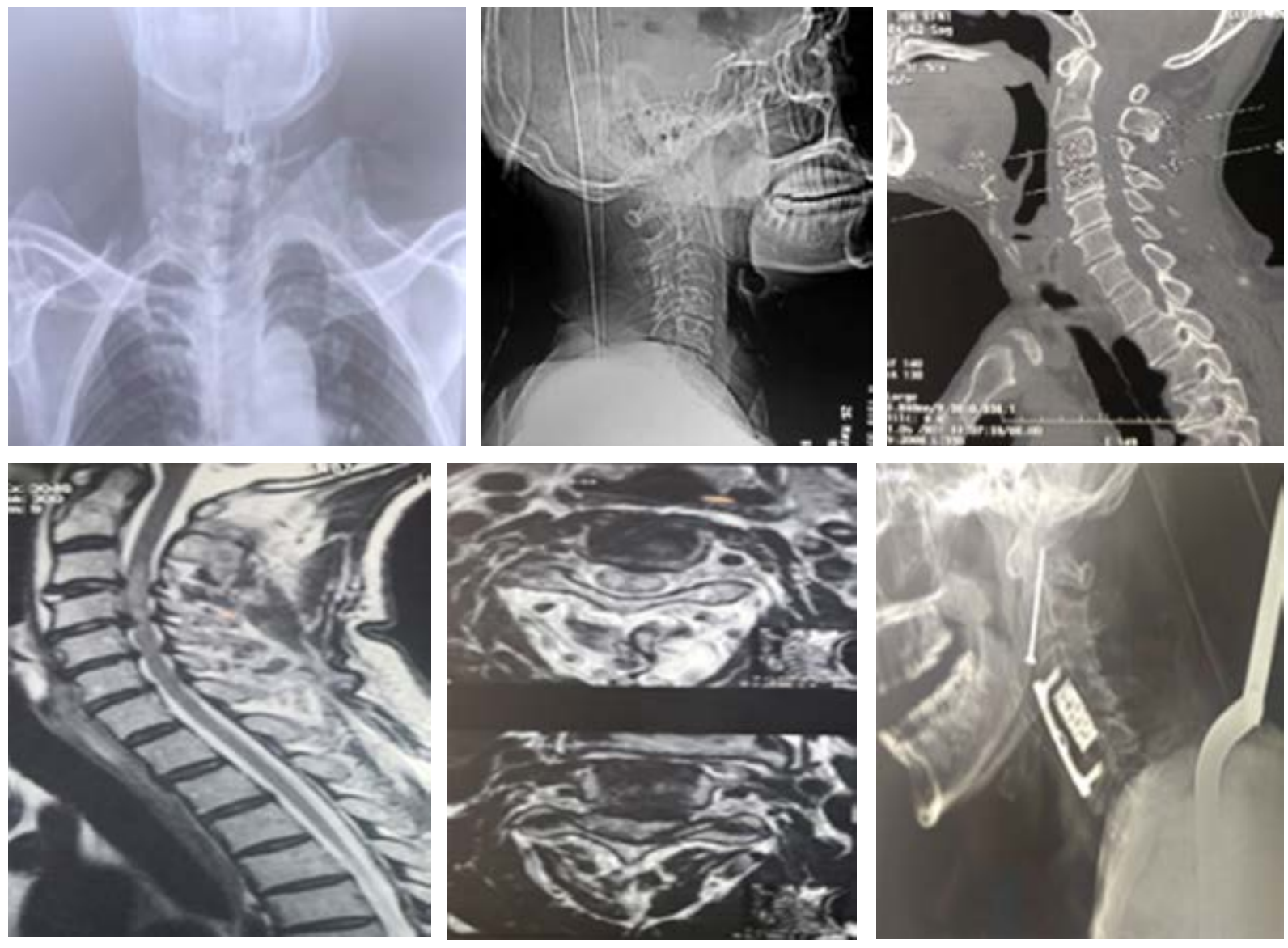

Figura 3: Paciente masculino de 65 años, mecanismo de alto impacto tras choque automovilístico, portaba cinturón de seguridad e iba como copiloto, presenta fractura de odontoides IIB con desplazamiento menor a $2 \mathrm{~mm}$ con presencia de radiculopatía y deterioro neurológico ASIA A por datos de mielopatía cervical crónica agudizada, por lo que se solicita resonancia magnética nuclear en la que se encuentran datos de mielopatía cervical en C3-C4, C4-C5, C5-C6. Se realiza manejo en un tiempo quirúrgico y abordaje anterior con reducción cerrada más colocación de tornillo odontoideo anterior más corpectomía C4 con hemicorpectomía C3 y C5 colocación de caja telescópica y placa cervical anterior.

\section{Resultados}

Se identificaron a 29 pacientes que cumplieron los criterios de inclusión, de los cuales 93.1\% (27 pacientes) fueron clasificados como Anderson y D'Alonzo tipo II y 6.9\% (dos pacientes) como Anderson y D'Alonzo tipo III. La edad promedio de los pacientes fue de $50.93 \pm 20.90$ años. El grupo de edad mayor a 65 años corresponde a $24.14 \%$ (siete pacientes); $55.2 \%$ de los pacientes fueron hombres (16 pacientes).

La cirugía fue el tratamiento primario en 20 pacientes (69\%), con abordaje anterior en $37.93 \%(11$ pacientes) y abordaje posterior en $31.03 \%$ (nueve pacientes). Por lo general, se prefirió la cirugía anterior en pacientes con líneas de fractura oblicua posterior u horizontal, dolor local y una superficie de contacto entre la odontoides y el cuerpo de C2. Se eligió un abordaje posterior cuando la distancia entre los fragmentos óseos fracturados fue $>2 \mathrm{~mm}$, conminución o que existiera alguna asociación que requiriera estabilización posterior Se realizó un abordaje 360 (tornillo cervical anterior e instrumentación posterior) en un paciente (3.45\%) que presentaba inestabilidad posterior además de una línea de fractura odontoidea horizontal.

La cinemática del trauma más frecuente fue la de alto impacto (caídas mayores de dos metros, accidentes de vehículo automotor), las cuales representan $75.9 \%$ (22 pacientes) de la población total, siendo $24.1 \%$ (siete pacientes) relacionados a mecanismos de bajo impacto. Sólo 13.64\% (4/29) 
Tabla 1: Frecuencias en las fracturas de apófisis odontoides tipo II y III Anderson y D'Alonzo.

\begin{tabular}{|c|c|c|c|}
\hline Datos demográficos (variables), $\mathrm{N}=29$ & $\mathrm{n}$ & $\begin{array}{l}\text { Frecuencia } \\
\text { relativa }\end{array}$ & $\%$ \\
\hline \multicolumn{4}{|l|}{ Sexo } \\
\hline Masculino & 16 & 0.552 & 55.2 \\
\hline Femenino & 13 & 0.448 & 44.8 \\
\hline \multicolumn{4}{|l|}{ Tratamiento (manejo) } \\
\hline Conservador & 9 & 0.3103 & 31.0 \\
\hline Anterior & 11 & 0.3793 & 38.0 \\
\hline Posterior & 9 & 0.3103 & 31.0 \\
\hline \multicolumn{4}{|l|}{$\begin{array}{l}\text { Clasificación de la fractura } \\
\text { (Anderson y D’Alonzo) }\end{array}$} \\
\hline Tipo II & 27 & 0.931 & 93.1 \\
\hline Tipo III & 2 & 0.069 & 6.9 \\
\hline \multicolumn{4}{|l|}{ Comorbilidades } \\
\hline TCE & 6 & 0.207 & 20.7 \\
\hline Mielopatía cervical & 3 & 0.103 & 10.3 \\
\hline Fractura cervical asociada & 8 & 0.276 & 27.6 \\
\hline Fractura toracolumbar & 3 & 0.103 & 10.3 \\
\hline Fractura otros huesos & 6 & 0.207 & 20.7 \\
\hline Otros & 4 & 0.138 & 13.8 \\
\hline \multicolumn{4}{|l|}{ Mecanismo de lesión } \\
\hline Hiperextensión & 22 & 0.759 & 75.9 \\
\hline Hiperflexión & 7 & 0.241 & 24.1 \\
\hline \multicolumn{4}{|l|}{ Cinemática del trauma } \\
\hline Alto impacto & 7 & 0.241 & 24.1 \\
\hline Bajo impacto & 22 & 0.759 & 75.9 \\
\hline \multicolumn{4}{|l|}{ Deterioro neurológico (ASIA) } \\
\hline$E$ & 22 & 0.760 & 76.0 \\
\hline$D$ & 1 & 0.034 & 3.4 \\
\hline C & 2 & 0.069 & 6.9 \\
\hline B & 1 & 0.034 & 3.4 \\
\hline$A$ & 1 & 0.034 & 3.4 \\
\hline No valorable & 2 & 0.069 & 6.9 \\
\hline \multicolumn{4}{|l|}{ Tiempo del tratamiento QX (días) } \\
\hline $3-7$ & 6 & 0.208 & 20.8 \\
\hline $7-14$ & 9 & 0.310 & 31.0 \\
\hline$>14$ & 5 & 0.172 & 17.2 \\
\hline No aplica (conservador) & 9 & 0.310 & 31.0 \\
\hline \multicolumn{4}{|l|}{ Consolidación } \\
\hline Adecuada & 16 & 0.552 & 55.2 \\
\hline Funcional & 3 & 0.103 & 10.3 \\
\hline Seudoartrosis & 5 & 0.172 & 17.2 \\
\hline No valorable (defunción) & 5 & 0.172 & 17.2 \\
\hline \multicolumn{4}{|l|}{ Complicaciones } \\
\hline Intubación prolongada & 8 & 0.276 & 27.6 \\
\hline Cervicalgia & 9 & 0.310 & 31.0 \\
\hline Neumonía & 5 & 0.172 & 17.2 \\
\hline Limitación funcional & 1 & 0.034 & 3.4 \\
\hline Muerte & 5 & 0.172 & 17.2 \\
\hline Ninguna & 8 & 0.276 & 27.6 \\
\hline
\end{tabular}

TCE = traumatismo craneoencefálico; ASIA = American Spinal Injury Association. de los pacientes con cinemática de alto impacto relacionada a la fractura fueron pacientes mayores de 65 años. El tiempo promedio transcurrido entre el accidente y el tratamiento definitivo fue de 14.1 días (de uno a 60 días).

El tratamiento conservador fue el tratamiento primario en nueve pacientes (31.03\%), utilizando principalmente collarín rígido tipo Philadelphia y el inmovilizador occipito-esternomandibular (collarín SOMI), así como en algunos casos el halo-chaleco. El tratamiento quirúrgico fue elegido como tratamiento en $68.97 \%$ (20 pacientes). Se realizó el abordaje anterior utilizando un tornillo odontoideo interfragmentario en nueve pacientes (31.03\%) (Figura 3). Se realizó una instrumentación posterior en $37.93 \%$ de los casos (10 pacientes). La mortalidad asociada fue de $17.2 \%$ (cinco pacientes) (Tabla 1).

En relación a la cinemática del trauma, la relacionada al mecanismo de alta energía en comparación con el de baja energía, cuenta con un OR de 27 veces mayor a tener algún tipo de daño que las lesiones de bajo impacto $(p<0.05)$. La edad mayor a 65 años presenta un riesgo mayor de daño de 8.4 veces que los menores de 65 años IC del 95\% 1.22-58.15 ( $p<0.05)$. De acuerdo con nuestro estudio no existe diferencia significativa entre el manejo conservador y el manejo quirúrgico según indicaciones respecto a la reducción adecuada OR 0.16 IC del 95\% 0.08-4.5, al igual que la consolidación con un OR 0.26 IC del 95\% 0.05-1.4.

Las complicaciones asociadas se presentaron tanto en la cinemática de alto y bajo impacto con un OR de 0.35 con un IC del 95\% de 0.03-3.35. La cinemática del trauma de alto impacto comparada con la de bajo impacto se asocia a un aumento de la mortalidad con un OR de 0.13 IC del 95\% 0.01-1.07. El tiempo de cirugía menor a siete días posteriores al accidente en los pacientes que se manejaron de

Tabla 2: Análisis de los factores de riesgo en las fracturas de apófisis odontoides tipo II Anderson y D'Alonzo.

\begin{tabular}{lcc}
\hline Variables asociadas & OR (IC del 95\%) & $\mathrm{p}$ (Fisher) \\
\hline Cinemática/consolidación & $27(2.5-291)$ & 0.02 \\
Edad/cinemática & $8.4(1.22-58.15)$ & 0.03 \\
Manejo/reducción & $0.16(0.08-4.5)$ & 0.11 \\
Manejo/consolidación & $0.26(0.05-1.4)$ & 0.05 \\
Complicaciones/cinemática & $0.35(0.03-3.35)$ & 0.35 \\
Cinemática/mortalidad & $0.13(0.01-1.07)$ & 0.07 \\
Tiempo Qx/mortalidad & $0.95(0.08-10.49)$ & 0.07 \\
\hline
\end{tabular}


forma quirúrgica se relacionó ligeramente como un factor protector relacionado con la mortalidad con un OR de 0.95 IC del 95\% 0.08-10.49 (Tabla 2).

\section{Discusión}

El tratamiento de las fracturas de odontoides está influenciado por una serie de factores como el tipo de fractura, la asociación con otras lesiones, la edad del paciente y las comorbilidades. ${ }^{7,9}$ La complicación más frecuente fue la cervicalgia, seguida de la intubación prolongada que conllevó a neumonía asociada a ventilador en $17.2 \%$ de los pacientes. El tratamiento quirúrgico fue el de elección en $68.97 \%$ (20 pacientes), siempre basándose en las características del paciente y el tipo de trazo de fractura. ${ }^{10,11}$

El manejo para las fracturas de odontoides tipo II se puede dividir en dos grandes grupos, conservador y quirúrgico, este último se divide en dos abordajes en general. ${ }^{12}$ La primera opción es el abordaje anterior con la colocación de uno o dos tornillos odontoideos interfragmentarios, el cual se describe como la técnica de elección en la mayoría de las fracturas Anderson y D'Alonzo tipo II, siendo en específico los subtipos de acuerdo con la clasificación de Grauer IIA y IIB. ${ }^{13}$ El manejo quirúrgico con abordaje posterior se debe realizar de la mano con una reducción cerrada previa colocación de un halo cefálico y al igual que el tornillo odontoideo, control imagenológico transquirúrgi$\mathrm{co}$, realizando la instrumentación posterior C1-C2 dependiendo del tipo de trazo y el desplazamiento que se presente. ${ }^{14}$ Ambos abordajes son un recurso que, si bien cuenta con indicaciones específicas, es a elección del cirujano quien realizará un abordaje anterior o tras la valoración integral del paciente optará por un abordaje posterior al presentar algún dato de inestabilidad, o que tras la osteosíntesis anterior pudiera presentar una insuficiencia de la reducción y estabilidad. ${ }^{15,16}$

El abordaje posterior es utilizado principalmente cuando el desplazamiento del fragmento hacia anterior o posterior es mayor de $6 \mathrm{~mm}$, presenta un desplazamiento mayor a $2 \mathrm{~mm}$ entre fragmentos 0 , cuando falla el tratamiento primario conservador 0 el tornillo odontoideo anterior. 7,17 Estudios previos realizan el análisis de las tasas de seudoartrosis con una correlación estadísticamente confiable sólo con la edad de los pacientes. ${ }^{14}$ Ninguna otra característica de la técnica quirúrgica tuvo un impacto significativo en la tasa de seudoartrosis. Uno de los factores más importantes que se correlaciona con la tasa de complicaciones relacionadas con la osteosíntesis anterior y posterior es la habilidad y el nivel de experiencia del cirujano. ${ }^{7,18} \mathrm{La}$ aplicación de navegación da como resultado una disminución de la exposición a la radiación y una precisión considerablemente mayor, por lo que la navegación es la alternativa adecuada para la fijación de la fractura odontoidea. ${ }^{14,19}$

Dentro de las limitaciones de nuestro estudio es operador dependiente, el criterio del cirujano es un factor imprescindible en la decisión del tratamiento quirúrgico, siempre guiándose de los criterios para la decisión del manejo, ya sea conservador o quirúrgico y el tipo de abordaje. ${ }^{20}$ La participación de varios cirujanos de columna es también una de las fortalezas, ya que cada uno de los pacientes es sometido a un consenso para valorar su manejo, más aún existe la posibilidad de variación en el tratamiento, inclusive en patrones de fractura similares siendo difícil controlar la indicación quirúrgica y la elección del tratamiento debido a los hallazgos en el paciente y la decisión del manejo. ${ }^{21,22}$ Nuestro estudio aporta la descripción de técnicas utilizadas en nuestro medio con un nivel de aplicación amplia y reproducible. En el análisis de la bibliografía existen claras tendencias que indican formas de mejorar los resultados quirúrgicos en pacientes con fracturas de odontoides, siendo el manejo principal en la actualidad. ${ }^{23,24}$

\section{Conclusiones}

El uso de las tecnologías adecuadas desde el control imagenológico transquirúrgico, del navegador y de tornillos canulados permitirán cada vez un avance en la cirugía de columna al poder ofrecer a un número mayor de pacientes un manejo óptimo, lo que llevará a la disminución de las complicaciones tanto en el manejo conservador como en el quirúrgico.

El tratamiento quirúrgico en los pacientes con fractura de odontoides con instrumentación posterior 0 tornillo interfragmentario anterior presentan un mayor índice de éxito en su resolución con dicha patología mas no modifica el índice de morbimortalidad asociada. El tratamiento de elección para las fracturas de un odontoides tipo II Anderson y D’Alonzo es la cirugía por abordaje anterior con el uso de uno o dos tornillos interfragmentarios de acuerdo con las características del trazo de la fractura, la reducción lograda y el tiempo de evolución al realizar el acto quirúrgico. El abordaje posterior con instrumentación C1-C2 es un manejo adecuado en las fracturas de odontoides con resultados 
favorables y de elección donde se clasifique una fractura tipo II Anderson y D'Alonzo subtipo C de Grauer o que se encuentre más de fragmento en el trazo odontoideo.

\section{Referencias}

1. AlEissa SI, Alhandi AA, Bugis AA, Alsalamah RK, Alshedd A, Almubarak AK, AIAssiri SS, Konbaz FM. The incidence of odontoid fractures following trauma in a major trauma center, a retrospective study. J Craniovert Jun Spine. 2020; 11: 71-74.

2. Marchesi DG. Current management of odontoid fractures. Indian Journal of Neurotrauma. 2005; 2: 3-6.

3. Gehweiler D, Wahnert D, Meier N, Spruit M, Raschke MJ, Richards RG, et al. Computational anatomy of the dens axis evaluated by quantitative computed tomography: Implications for anterior screw fixation. J Orthop Res. 2017; 35 (10): 21542163.

4. Terrence D. Julien, Bruce Frankel, Vincent C. Traynelis, Timothy C. Ryken. Evidence-based analysis of odontoid fracture management. Neurosurg Focus. 2000; 8 (6): 1-6.

5. Tekdemir I. Anatomical study of axis for odontoid screw thickness, length and angle. Eur Spine J. 2009; 18: 271-275.

6. Shousha M, Alhashash M, Allouch H, Boehm H. Surgical treatment of type II odontoid fractures in elderly patients: a comparison of anterior odontoid screw fixation and posterior atlantoaxial fusion using the Magerl-Gallie technique. Eur Spine J. 2019; 3-9.

7. Falavigna A, Righesso O, da Silva PG, Siri CR, Daniel JW, Esteves Veiga JC, et al. Management of type II odontoid fractures: experience from latin american spine centers. World Neurosurg. 2017; 98: 673-681.

8. Maak TG, Grauer JN. The contemporary treatment of odontoid injuries. Acta Orthop. Belg. 2018; 84: 108-115.

9. Watanabe M, Sakai D, Yamamoto Y. Analysis of predisposing factors in elderly people with type II odontoid fracture. Spine $J$. 2013; 14 (6): 861-866.

10. Carvalho AD, Figueiredo J, Schroeder GD, Vaccaro AR, Rodrigues-Pinto R. Odontoid fractures: a critical review of current management and future directions. Clin Spine Surg. 2019; 32 (8): 313-323.

11. Iyer S, Hurlbert RJ, Albert TJ. Management of odontoid fractures in the elderly: a review of the literature and an evidence-based treatment algorithm. Neurosurgery. 2018; 82 (4): 419-430.

12. Hubner AR, De Freitas Spinelli L, Magadiel Klaus A. Decisión en el tratamiento de las fracturas del odontoides. Coluna/ Columna. 2010; 9 (1): 43-48.

13. Joestl J, Lang N, Bukaty A, Platzer P. A comparison of anterior screw fixation and halo immobilisation of type II odontoid fractures in elderly patients at increased risk from anaesthesia. Bone Jt J. 2016; 98-B (9): 1222-1226.

14. Lvov I, Grin A, Talypov A, Godkov I, Kordonskiy A, Khushnazarov $\mathrm{U}$, et al. The impact of odontoid screw fixation techniques on screw-related complications and fusion rates: a systematic review and meta-analysis. Eur Spine J. 2020; 6: 6501-6509.

15. Abolfotouh S, Moore D. Use of simultaneous traction over a halo ring to achieve reduction of a type 2 odontoid fracture for anterior odontoid screw fixation. Int J Surg Case Rep. 2019; 64: 97-101.

16. Mohamed M, A Hewedy M. Anterior Trans-odontoid Screw Fixation in Odontoid Fractures. Egypt Spine J. 2017; 22 (1): 41-49.

17. Rao G, Apfelbaum RI. Odontoid screw fixation for fresh and remote fractures. Neurology India. 2005; 53 (4): 416-423.

18. Wang LN, Li T, Yang X, Wang L, Liu LM, Liu H, et al. Comparison of two temporary fixation techniques for the treatment of type II odontoid fracture. Acta Orthop Belg. 2018; 84 (1): 108-115.

19. Tang S. C-1 lateral mass screw. J Neurosurg Pediatr. 2010; 6 (5): 513-514.

20. Joaquim AF, Patel AA. Specific considerations in older patients. Neurosurg Focus. 2015; 38: 1-6.

21. Charles YP, Ntilikina Y, Blondel B, Fuentes S, Allia J, Bronsard $\mathrm{N}$, et al. Mortality, complication, and fusion rates of patients with odontoid fracture: the impact of age and comorbidities in 204 cases. Arch Orthop Trauma Surg. 2019; 139 (1): 43-51.

22. Yang Z, Yuan ZZ, Ma JX, Ma XL. Conservative versus surgical treatment for type II odontoid fractures in the elderly: grading the evidence through a meta-analysis. Orthop Traumatol Surg Res. 2015; 101 (7): 839-844.

23. Baogui L, Juwen C. Fusion rates for odontoid fractures after treatment by anterior odontoid screw versus posterior C1-C2 arthrodesis?: a meta-analysis. Arch Orthop Trauma Surg. 2019; 139 (10): 1329-1337.

24. Konieczny MR, Gstrein A, Müller EJ. Treatment algorithm for dens fractures: non-halo immobilization, anterior screw fixation, or posterior transarticular C1-C2 fixation. J Bone Joint Surg Am. 2012; 94 (19): 1-6.

\section{Conflicto de intereses}

El autor refiere no presentar algún conflicto de intereses con la presente investigación. 